\title{
Avaliação do equilíbrio, postura e qualidade de vida de deficientes visuais
}

\author{
Evaluation of balance, posture e and quality of life of the visually impaired
}

\author{
Gabriela Gonçalves Machado ${ }^{1, *}$ \\ Izadira Cristina Barcelos Oliveira ${ }^{1}$ \\ Wilbert Esteban Cárdenas Urquizo ${ }^{1}$ \\ Suraya Gomes Novais Shimano ${ }^{1}$ \\ Nuno Miguel Lopes Oliveira ${ }^{1}$
}

\section{Resumo}

Objetivo:Avaliar o equilíbrio, a postura e a qualidade de vida (QV) de pessoas com deficiência visual (DV) que não praticam atividade física (AF). Métodos: Participaram do estudo 8 pessoas com DV. Critérios de inclusão: apresentar DV, ser cadastrado no Instituto dos Cegos do Brasil Central e aceitar participar. Critérios de não inclusão: deficiência auditiva ou sensorial, déficit cognitivo, labirintite e realizar AF. Foram excluídos os que não foram em todas as avaliações. Foi avaliado o equilíbrio pela baropodometria, a postura pelo Software de Avaliação Postural (SAPO) e QV pelo questionário World Health Organization Qualityof Life Instruments - Bref. Foi realizada a análise descritiva de todos os dados, os baropodométricos e do SAPO foram submetidos ao teste de Wilcoxon. Resultados: Houve uma oscilação ântero-posterior (AP) significativamente maior em relação a látero-lateral em apoio unipodal $(A U)$ direito $(p=0,01)$ e AU esquerdo $(p=0,01)$. No SAPO as alterações significativas foram o ângulo entre os dois acrômios e as duas espinhas ilíacas ântero-superiores $(p=0,03)$, alinhamento vertical da cabeça direito $(p=0,04)$ e esquerdo $(p=0,02)$. Na QV apresentou menores escores nos domínios ambiente $55,47 \%$ e relações socias 60,42\%. Conclusão: Os DV não praticantes de AF apresentam uma oscilação corporal significativamente maior no sentido AP, anteriorização da cabeça, protrusão de ombros, anteroversão pélvica e baixos escores de QV.
\end{abstract}

Palavras-chave: deficiência visual, equilíbrio postural, postura, qualidade de vida.

\begin{abstract}
Objective: To evaluate the balance, posture and quality of life (QF) of visual impairment (VI) people who don't practice physical activities (PA). Methods: Participated in thestudy 8 people with VI. Inclusion criterion: be registered as VI people on the Instituto dos Cegos do Brasil Central and agree to participate. Non-inclusion criterion: to have hearing or sensory impairment, cognitive deficits, labyrinthitis or practice PA. Exclusion criteria was not attending in allthe evaluations. The balance was evaluated by baropodometry, posture by the Postural Evaluation Software (SAPO) and QF was based on the questionnaire World Health Organization Quality of Life Instruments - Bref. It was made a descriptive analysis of the data, the baropodometric and the SAPO were submitted to the Wilcoxon. Test. Results: There was a bigger oscilation anteroposterior (AP) in relation of latero lateral in right unipodal support (US) and left US $(p=0.01)$. In SAPO the significant changes were the angle between the two acromion and the two anterior superior iliacspines $(p=0.03)$, vertical alignment of the righthead $(p=0.04)$ andleft $(p=0.02)$. In the QF presented lower scores in the environment domains $55.47 \%$ and social relationships $60.42 \%$. Conclusion: The VI non-practitioners of PA have a significantly greater body oscillation in thesense AP, anterior head, shoulder protrusion, pelvic anteroversion and low AF scores.
\end{abstract}

Keywords: vision disorders, postural balance, posture, quality of life.
Afiliação dos autores

Universidade Federal Do Triângulo Mineiro, Uberaba, Minas Gerais, Brasil.

\section{${ }^{*}$ Autor correspondente}

Departamento de Fisioterapia Aplicada, Rua Vigário Carlos, 100, Bloco B, 4ำandar, Sala 406, Abadia CEP: 38025-350, Uberaba, Minas Gerais, Brasil.

e-mail:

glaabi_gmachado@hotmail.com

Conflito de interesses

Os autores declararam não haver conflito de interesses.

Processo de arbitragem

Recebido: 29/12/2018 Aprovado: 04/03/2019 


\section{Introdução}

A deficiência visual (DV) caracteriza-se por perdas totais ou parciais da visão em decorrência a causas hereditárias, congênitas ou adquiridas, onde mesmo após correções óticas ou cirúrgicas, há limitações de seu desempenho normal ${ }^{1}$. Ela engloba a baixa visão ou cegueira e os indivíduos apresentam comprometimento funcional e dificuldades na realização de tarefas $^{2}$.

Estima-se que $1 \%$ da população mundial apresenta algum grau de deficiência visual e que mais de $90 \%$ são em países em desenvolvimento. No Brasil, segundo dados de 2010, existem mais de 6,5 milhões de pessoas com DV, sendo 6 milhões com baixa visão e 582 mil com cegueira total ${ }^{3}$.

A DV acarreta várias incapacidades e, quando são associadas à carência de acessibilidade de espaços públicos e particulares, ocasionam a não inclusão na sociedade e falta de autonomia nas suas atividades de vida diária, provocando à pessoa com DV uma redução da sua qualidade de vida ${ }^{4}$.

Cabe ressaltar que a visão exerce uma importante função na estabilidade postural dos indivíduos, a qual é a capacidade de se restaurar ou reestabelecer o equilíbrio corporal independente do movimento e posição do corpo e, na pessoa com DV, o sistema visual, um dos três sistemas responsáveis pelo controle da estabilidade postural, está ausente ou alterado. Com isso, há uma perda funcional dos mecanismos visuais relacionados ao controle de equilíbrio, o que acarreta um equilíbrio prejudicado, tanto em tarefas estáticas quanto dinâmica ${ }^{5,6}$.

A manutenção do equilíbrio, estático ou dinâmico, depende da integração e processamento de sistemas do Sistema Nervoso Central ligados ao equilíbrio, bem como área de apoio, posicionamento dos pés, características musculares e restrições das tarefas ${ }^{1,7}$. Uma vez que a visão exerce um papel importante no equilíbrio do indivíduo, o seu déficit provoca posturas compensatórias e alterações posturais ${ }^{8,9}$, no intuito de reorganizar o seu corpo, ambientá-lo ao espaço e manter o seu equilíbrio ${ }^{10}$.

Em decorrência das alterações de postura e equilíbrio, a pessoa com DV tem maior tendência a quedas, gerando uma conduta de reclusão por parte do indivíduo. Esta atitude de sedentarismo pode resultar em uma diminuição de impulsos nervosos e alterações fisiológicas importantes para o controle do corpo e mente ${ }^{11}$.

Estudos mostram a importância da atividade física no equilíbrio, visto que exercícios usados para ganho de massa muscular, força e resistência, melhoram o equilíbrio e reduzem o risco de quedas, além de contribuir para a qualidade de vida ${ }^{12}$. pessoa com DV que não pratica atividade física torna-se mais vulnerável às alterações de equilíbrio, da postura e da qualidade de vida, uma vez que a atividade físicaé uma estratégia utilizada para proporcionar um maior grau de independência e segurança ${ }^{13}$. Estudos com essa população mostraram que a prática de exercício físico auxilia no ganho de equilíbrio ${ }^{14,15}$, na qualidade de vida e na melhora da postura ${ }^{16}$.

Tendo em vista a susceptibilidade das pessoas com DV a apresentar alterações posturais, de equilíbrio, da qualidade de vida e uma possível relação de melhora dessas variáveis perante a prática de atividade física, é importante avaliar essas alterações para se ter o conhecimento das compensações corporais mais frequentes e das necessidades individuais para assim subsidiar profissionais nas prescrições de exercícios e intervenções. Diante do exposto, o objetivo do estudo é avaliar a postura, o equilíbrio e a qualidade de vida de pessoas com DV que não praticam atividade física.

\section{Métodos}

\section{Desenho do Estudo}

Estudo observacional de corte transversal, com abordagem quali-quantitativa.

\section{Local do Estudo}

Foi realizado no Instituto dos Cegos do Brasil Central (ICBC) de Uberaba, Minas Gerais, o qual é centro de referência em atendimentos de pessoas com DV de toda essa região.

Comitê de Ética em Pesquisa
O presente estudo foi aprovado pelo Comitê de Ética em Pesquisa da Universidade Federal do Triângulo Mineiro (UFTM), sob o parecer do número 2.151.596.

\section{Voluntários}

Os critérios de inclusão foram ser cadastrados no ICBC, apresentar interesse, disponibilidade e aceitar em participar da pesquisa, assinando $o$ termo de consentimento livre $e$ esclarecido (TCLE), o qual foi lido por um único avaliador. O TCLE não foi disponibilizado em braile pois alguns voluntários não sabiam ler.Os critérios de não inclusão foram apresentar deficiência auditiva ou sensorial, déficit cognitivo, labirintite e estar realizando alguma atividade física. O critério de exclusão foi não comparecer em todas as avaliações.

A partir desses critérios, a amostra foi composta por oito pessoas com DV, de ambos os sexos, sendo $5 \mathrm{com}$ baixa visão e 3 com cegueira total. A confirmação do grau da deficiência visual se deu por meio da busca de prontuários médicos disponibilizados no ICBC.

\section{Avaliações}

Pessoas com DV são privadas de diversas informações ao seu redor e, para compensar essa ausência visual, são necessárias algumas adaptações durante as tarefas, bem como nas avaliações, com o propósito de estimular os demais sistemas preservados. Assim, em todas as avaliações foram realizadas explicações sobre cada etapa dos procedimentos, a fim de manter o indivíduo ciente do ambiente e da situação para diminuir o medo do desconhecido.

Foi avaliado o equilíbrio por meio da baropodometria, utilizando o baropodômetro eletrônico T.plate(Medicapteurs, França). Os voluntários foram posicionados de forma estática em posição ortostática de três modos: apoio bipodal; apoio unipodal direito e unipodal esquerdo com olhos abertos. Em cada posicionamento foram realizadas quatro coletas, com duração de 30 segundos, sendo a primeira para teste e calibração do equipamento. O tempo de descanso entre cada avaliação foi de 30 segundos.

Os voluntários deveriam estar descalços, a fim de minimizar a influência de diferentes tipos de calçados. Os pés foram posicionados à largura do quadril, com os braços alinhados ao lado do corpo e os voluntários orientados a manterem o olhar em sentido ao horizonte. De acordo com a padronização de Cárdenas-Urquizo ${ }^{15}$ na avaliação de apoio bipodal, o voluntário foi posicionado sobre a plataforma com os retropés em uma distância de $10 \mathrm{~cm}$ e o restante dos pés livres. Na avaliação de apoio unipodal direito e esquerdo, o voluntário era posicionado sobre a plataforma com o pé direito ou esquerdo no centro. Foram repassadas algumas orientações aos voluntários, devido à deficiência visual, para que realizassem os testes com segurança: orientação sobre a presença de uma cadeira posicionada posteriormente a eles, sobre a presença de uma barra de ferro de apoio, de uma parede na sua lateral direita e de uma pessoa a sua frente para fornecer suporte em casos de desequilíbrios (Figura 1A).
A)

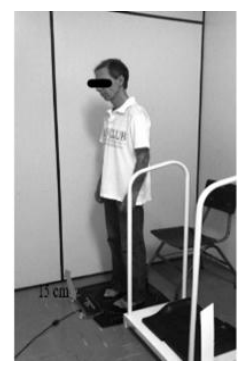

B)

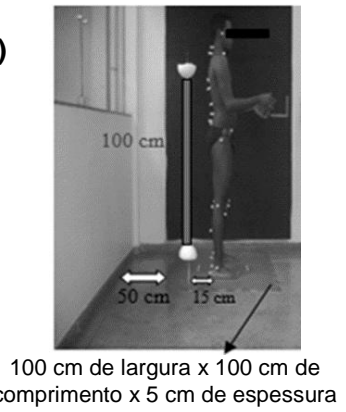

Figura 1. A) Local de realização da baropodometria com a presença da barra de apoio, cadeira e parede a $15 \mathrm{~cm}$ do equipamento. B) Representação esquemática da avaliação postural na vista lateral direita, demonstrando o tablado de EVA, o fio de prumo e as distâncias dos posicionamentos.

A avaliação postural foi realizada por biofotogametria analisada pelo Software de Avaliação Postural (SAPO). Foram 
utilizados marcadores esféricos acoplados à pele, em pontos anatômicos determinados. Os voluntários foram orientados a utilizar trajes de banho para melhor demarcação dos pontos, os quais foram: trago da orelha (TO); acrômio (AC); espinha ilíaca ântero-superior (EIAS); espinha ilíaca póstero-superior (EIPS); trocânter maior do fêmur (TF); ponto medial da patela (MP); tuberosidade da tíbia (TT); ângulo inferior da escápula (AIE); linha articular do joelho (LAJ); ponto sobre a linha média da perna (LMP); ponto sobre o tendão de Aquiles na altura média dos dois maléolos (TA) e calcâneo (C); maléolo lateral e medial ( $\mathrm{ML}$ e $\mathrm{MM})$; quarta vértebra cervical (C4); sétima vértebra cervical (C7); sétima vértebra torácica (T7); décima segunda vértebra torácica (T12); terceira vértebra lombar (L3); quinta vértebra lombar (L5). Todos de forma bilateral, à exceção dos segmentos da coluna vertebral ${ }^{17}$

As imagens foram capturadas em quatro vistas: anterior, posterior, lateral direita e esquerda. Os voluntários foram posicionados pelo avaliador por meio de comandos táteis e verbais em relação ao posicionamento dos pés, braços e distância do fio de prumo. Foram orientados a manter os pés em paralelo sobre o tablado de EVA $(100 \mathrm{~cm}$ de largura $\times 100 \mathrm{~cm}$ de comprimento $\times 5 \mathrm{~cm}$ de espessura), que estava a uma distância de $50 \mathrm{~cm}$ da parede lateral direita e encostado na parede posterior. Nas vistas laterais, o voluntário ficou a $15 \mathrm{~cm}$ do fio de prumo $^{18}$. Foi usada uma câmera digital Cyber-shot 7.2 megapixels (SONY, Brasil), a três metros dos voluntários, em um tripé, posicionada a $100 \mathrm{~cm}$ do solo (Figura 1-B).

$A$ qualidade de vida foi avaliada pelo questionário auto aplicável WHOQOL-BREF, preconizado pela Organização Mundial de Saúde e validado, no Brasil, por Fleck ${ }^{19}$. O questionário conta com 26 questões, sendo duas para qualidade de vida geral e as 24 questões são divididas em facetas, as quais compõem os domínios físico, psicológico, relações sociais e ambiente. A aplicação do questionário se deu por meio da leitura por um mesmo avaliador, em uma sala vazia, para maior liberdade do voluntário.

\section{Análise Estatística}

A análise estatística dos dados foi realizada no software Statistical Package for the Social Sciences (SPSS), versão 22.0. Inicialmente todos os dados foram analisados de forma descritiva.

Para as análises baropodométricas, foi descartada a primeira coleta feita em cada posicionamento e realizada a média entre as três coletas posteriores. A normalidade dos dados baropodométricos e do SAPO foram verificados por meio do teste Shapiro-Wilk. Posteriormentefoiaplicado o teste de Wilcoxon para duas amostras relacionadas comparando as varáveis baropodométricas: amplitude ântero-posterior e amplitude látero-lateral em apoio bipodal, apoio unipodal direito, apoio unipodal esquerdo, área de apoio unipodal direito e área de apoio unipodal esquerdo. No SAPO, as variáveis foram comparadas com os seus respectivos valores de referência. Foi considerado o valor de $\mathrm{p} \leq 0,05$ para todas as análises.

\section{Resultados}

A amostra foi composta por oito indivíduos com DV, sendo quatro do sexo feminino (todas com baixa visão) e quatro do sexo masculino (sendo dois com baixa visão e dois com cegueira total). Na Tabela 1 encontram-se os dados descritivos da amostra.

Tabela 1

Análise descritiva da amostra.

\begin{tabular}{lc}
\hline \multirow{2}{*}{ Variável } & Amostra $(\mathrm{n}=8)$ \\
\cline { 2 - 2 } & Média \pm Desvio Padrão \\
\hline Idade $($ anos $)$ & $38,38 \pm 11,67$ \\
Estatura $(\mathrm{m})$ & $1,65 \pm 0,11$ \\
IMC $\left(\mathrm{kg} / \mathrm{m}^{2}\right)$ & $27,65 \pm 7,56$ \\
Massa corporal $(\mathrm{Kg})$ & $73,78 \pm 17,68$ \\
Tamanho do pé $(\mathrm{cm})$ & $24,06 \pm 1,63$ \\
\hline IMC: Indice de massa corporal.
\end{tabular}

$\mathrm{Na}$ avaliação de equilíbrio foi possível observar por meio das variáveis baropodométricas uma oscilação do centro de pressão ântero-posterior significativamente maior em relação à oscilação látero-lateral, nas posições apoio unipodal direito e apoio unipodal esquerdo. Apresentaram uma maior área de apoio unipodal direito em relação apoio unipodal esquerdo, porém não foi significativo. Na Tabela 2 estão descritos os dados de cada variável em média, desvio padrão e o valor de $p$ da área de apoio unipodal direito em relação a área de apoio unipodal esquerdo, amplitude látero-lateral em relação a amplitude ântero-posterior nos apoios bipodal, unipodal direito, unipodal esquerdo, respectivamente.

Tabela 2

Variáveis baropodométricas.

\begin{tabular}{ccc}
\hline Variável & Média e DP & p-valor \\
\hline Área AB $\left(\mathrm{mm}^{2}\right)$ & $62,92 \pm 49,97$ & \\
Área AUD $\left(\mathrm{mm}^{2}\right)$ & $537,19 \pm 439,18$ & 0,33 \\
Área AUE $\left(\mathrm{mm}^{2}\right)$ & $424,93 \pm 302,77$ & \\
Amp LL AB $(\mathrm{mm})$ & $7,37 \pm 4,32$ & 0,12 \\
Amp AP AB $(\mathrm{mm})$ & $9,96 \pm 4,64$ & \\
Amp LL AUD $(\mathrm{mm})$ & $17,29 \pm 6,48$ & $0,01^{*}$ \\
Amp AP AUD $(\mathrm{mm})$ & $33,02 \pm 17,00$ & \\
Amp LL AUE $(\mathrm{mm})$ & $16,11 \pm 6,77$ & $0,01^{*}$ \\
Amp AP AUE $(\mathrm{mm})$ & $30,07 \pm 13,97$ & \\
\hline DP: Desvio padrão; AB: Apoio Bipodal; AUD: Apoio Unipodal Direito; AUE:
\end{tabular}
Apoio Unipodal Esquerdo; Amp: Amplitude; LL: Látero-lateral; AP: Ânteroposterior; ${ }^{*} p<0,05$.

$\mathrm{Na}$ avaliação postural, foi observado que todas as variáveis apresentaram valores diferentes aos de referência do SAPO ${ }^{20}$ estes que não são específicos para a população deficiente visual, porém essa diferença foi significativa apenas para o ângulo entre os dois acrômios e as duas espinhas ilíacas ânterosuperiores, alinhamento vertical da cabeça direto e esquerdo. Todas as variáveis são apresentadas na tabela 3, por média, desvio padrão, valores de referência e p-valor entre as médias das variáveis e seus respectivos valores de referência.

Tabela 3

Variáveis do SAPO

\begin{tabular}{|c|c|c|c|}
\hline Variável & Média e DP & Referência & p-valor \\
\hline $\mathrm{AHC}\left({ }^{\circ}\right)$ & $0,77 \pm 3,11$ & $0^{\circ}$ & 0,67 \\
\hline $\mathrm{AHA}(\stackrel{\circ}{)})$ & $-1,92 \pm 2,50$ & $0^{\circ}$ & 0,07 \\
\hline AHEIAS $(\stackrel{\circ}{\circ})$ & $1,84 \pm 2,77$ & $0^{\circ}$ & 0,14 \\
\hline AAEIAS $(\stackrel{\circ}{\circ})$ & $3,75 \pm 40,26$ & $0^{\circ}$ & $0,03^{*}$ \\
\hline DCMMII (cm) & $0,10 \pm 3,28$ & $0 \mathrm{~cm}$ & 0,16 \\
\hline AHTT (ํ) & $-0,21 \pm 1,68$ & $0^{\circ}$ & 1,00 \\
\hline$A Q D(\stackrel{\circ}{)}$ & $21,95 \pm 12,05$ & $15^{\circ}$ & 0,09 \\
\hline AQE $(\because)$ & $17,75 \pm 10,65$ & $15^{\circ}$ & 0,57 \\
\hline $\operatorname{AVCD}(\stackrel{\circ}{\circ})$ & $18,29 \pm 21,72$ & $0^{\circ}$ & $0,04^{*}$ \\
\hline AVCE $(\stackrel{\circ}{\circ})$ & $18,25 \pm 19,06$ & $0^{\circ}$ & $0,02^{*}$ \\
\hline
\end{tabular}

Alinhamento Horizontal dos Acrômios; (AHEIAS) Alinhamento Horizontal das Espinhas llíacas Ântero-superiores; (AAEIAS) Ângulo entre os dois acrômios e as duas espinhas ilíacas ântero-superiores; (DCMMII) Diferença no comprimento dos membros inferiores; (AHTT) Alinhamento horizontal das tuberosidades das tíbias; (AQD) Ângulo Q direito; (AQE) Ângulo Q esquerdo; (AVCD) Alinhamento vertical da cabeça direito; (AVCE) Alinhamento vertical da cabeça esquerdo. * $\mathrm{p}<0,05$.

A análise da qualidade de vida geral apontou um escore de $59,98 \%$ e os domínios com menor escore foram o ambiente $(55,47 \%)$ e relações sociais $(60,42 \%)$, respectivamente. Todos os domínios são apresentados na Figura 2.

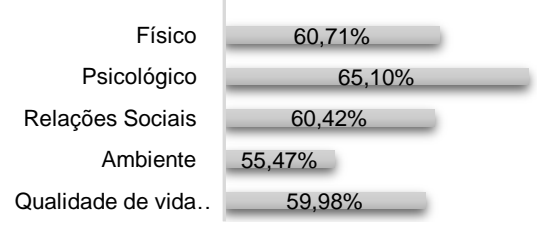

Figura 2. Escores dos domínios do Whoqol-Bref.

\section{Discussão}

O presente estudo teve como proposito uma análise qualiquantitiva do equilíbrio, postura e qualidade de vida de deficientes visuais não praticantes de atividade física.

$\mathrm{Na}$ análise do equilíbrio estático, foi possível observar uma maior oscilação do centro de pressão no sentido ânteroposteriorem relação ao látero-lateral, o que vai de encontro com a pesquisa de Cárdenas-Urquizo ${ }^{15}$, a qual também revelou um maior deslocamento ântero-posteriorantes e depois da realização de um protocolo de Pilates adaptado para pessoas com DV. A acuidade visual é um mecanismo importante para o 
controle postural, com isso, sua diminuição pode ser responsável pelo aumento da oscilação ântero-posterior ${ }^{9}$.

Nossa amostra apresentou uma média de IMC de 27,65 $\mathrm{Kg} / \mathrm{m}^{2}$, indicando sobrepeso e, de acordo com a literatura, existe correlação entre aumento de peso e instabilidade postural, pois são necessários maiores ajustes posturais para manter-se em equilíbrio ${ }^{21}$. Assim, além da restrição visual, ainda há a influência da massa corporal nos ajustes posturais da população avaliada.

Cabe ressaltar que, durante a manutenção de uma posição estática, há forças atuando de forma variada nos segmentos corporais, o que demonstra que um corpo nunca está totalmente imóvel. O centro de massa pode oscilar no sentido látero-lateral e ântero-posterior e, de acordo com Barela ${ }^{22}$, quando se priva a informação visual, tanto por olhos fechados ou por diminuição da acuidade visual, a oscilação corporal aumenta em um dos sentidos.

$\mathrm{Na}$ avaliação postural foram observados valores significativamente superiores aos de referência para indivíduos videntes nas variáveis do ângulo entre os dois acrômios e as duas espinhas ilíacas ântero-superiores, no alinhamento vertical da cabeça direto e esquerdo demonstrando que o indivíduo com a ausência da visão realiza compensações musculoesqueléticas para reorganizar o seu corpo no espaço e manter-se em equilíbrio. Dentre as alterações corpóreas foi observado anteriorização da cabeça, protrusão de ombros e anteroversão pélvica, o que corrobora com os achados dos estudos de Silva ${ }^{6}$, Catanzariti ${ }^{10}$ e Sanchez ${ }^{23}$

Os resultados dessa amostra apresentam diversidade de posicionamento dos segmentos corporais, provavelmente em função de ser um grupo heterogêneo. Deste modo, gera-se altos desvios que também podem interferir na interpretação dos achados, não oferecendo sustentação para a hipótese de um padrão de anormalidade de simetria postural ${ }^{24}$

$\mathrm{Na}$ avaliação da qualidade de vida, considera-se um escore total de $100 \%$ e, quanto mais próximo a esse valor, melhor o índice de qualidade de vida. De acordo com os resultados apresentados no estudo, os domínios meio ambiente e relações sociais apresentaram um escore mais prejudicado. Esses domínios estão relacionados com o ambiente físico, acesso a lugares públicos, segurança física, proteção, transporte, relações pessoais e suporte social ${ }^{19}$ Nossos achados corroboram com o estudo de Marques et al. ${ }^{11}$, o qual investigou os benefícios da Fisioterapia aquática no índice de qualidade de vida de pessoas com DV

Rebouças e colaboradores ${ }^{25}$ também obtiveram um baixo escore no domínio ambiental ao avaliar a qualidade de vida de pessoas com DV. Muitos estudos que utilizam o Whoqol-Bref em outras populações têm demonstrado que o domínio do meio ambiente é considerado como o mais prejudicado ${ }^{26,27}$. De acordo com Awasthi e seus colaboradores ${ }^{27}$, um escore baixo no domínio ambiente pode ser decorrente a uma baixa percepção do real pelo indivíduo quando comparado com a sua alta expectativa de ambientes e disponibilidade de vários fatores em sua vida, gerando inadequações entre o real e o esperando, levando a uma menor percepção de qualidade ambiental.

Andrade e colaboradores ${ }^{28}$ e Othero ${ }^{29}$ apontam a falta de infraestrutura adaptada para pessoas com deficiência como fator de restrição a acessos a lazer, cultura, transporte e empregos, além de ser uma possível explicação para um baixo resultado no âmbito ambiental. Segundo Haegele et $\mathrm{al}^{30}$, fatores pessoais como a dependência de outros indivíduos e também fatores ambientais, como transporte e dependência para locomoção, são barreiras que influenciam a prática de atividade física por pessoas com DV, podendo então afetar a sua qualidade de vida.

Deste modo, a pessoa com DV, que já é vulnerável diante de sua perda visual e das consequentes alterações corporais decorrentes a ela, bem aliado à a falta de acessibilidade, a qual dificulta o seu lazer e a prática de atividade de física, podem ser induzidos, perante essas limitações, ao sedentarismo e privação de lazer, gerando um reflexo negativo na sua qualidade de vida. Uma limitação do nosso estudo foi uma amostra pequena, devido ao fato de que as pessoas com DV cadastradas no ICBC eram de diferentes regiões, o que dificultava 0 seu deslocamento para as avaliações. Além disso, há uma alta heterogeneidade dos casos decorrentes a outras doenças associadas à deficiência visual.
Conclui-se que os DV não praticantes de atividade física apresentam uma oscilação corporal significativamente maior no sentido ântero-posterior, anteriorização da cabeça, protrusão de ombros, anteroversão pélvica e baixos índices de qualidade de vida, destacando os domínios de relações sociais e ambiente.

\section{Referências}

1. Santos ACS. Desempenho de criancas e adolescentes com baixa visão na Escala de Equilíbrio Pediátrica. [Dissertação de mestrado - Programa De Pós-Graduação em Saúde da Criança e do Adolescente]. Recife (PE): Universidade Federal de Pernambuco; 2016

2. Brasil. Ministério da Saúde. Gabinete do Ministro. Portaria no 3.128, de 24 de dez de 2008. Define que as Redes Estaduais de Atenção à Pessoa com Deficiência Visual sejam compostas por ações na atenção básica e Serviços de Reabilitação Visual [portaria na internet]. Diário Oficial da União $24 \mathrm{dez} 2008$ [acesso em $07 \mathrm{fev}$ 2018].Disponível em: http://bvsms.saude.gov.br/bvs/saudelegis/gm/2008/prt3128_24_12_2008. html.

3. Oliveira DG, Shimano SGN, Salomão AE, Pereira K. Avaliação do perfil socioeconômico, formação profissional e estado de saúde de pessoas com deficiência visual. RevBrasOftalmol 2017; 76 (5): 255-258.

4. Frade MCM Cardeña JP Shimano SGN, Oliveira CCES, Oliveira NML.Equilíbrio dos deficientes visuais antes e após gameterapia.RevEducEsp 2014; 27(50): 751-764.

5. Parreira RB, Grecco LAC, Oliveira CS. Postural control in blind individuals: A systematic review. Gait Posture2017;57:161-167.

6. Silva MB, Shimano SGN, Oliveira CCES, Conti V, Oliveira NML. Avaliação das alterações posturais e retrações musculares na deficiência visual: estudo de caso. Saúde Colet 2011; 8(49):77-82.

7. Ruhe A, Fejer R, Walker B. The test-retest reliability of center of pressure measures in bipedal static task conditions - A systematic review of the literature. GaitPosture 2010;32:436-445.

8. Friedrich M, Grein HJ, Wicher C, Schuetze J, Mueller A, Lauenroth A Hottenrott K, Schwesig R. Influence of pathologic and simulated visual dysfunctions on the postural system. Exp Brain Res 2008; 186(2):305314

9. Oliveira DN, Barretos RR. Avaliação do equilíbrio estático em deficientes visuais adquiridos. RevNeurocienc 2015; 13(3):122-127.

10. Catanzariti JF, Salomez E, Bruandet JM, Thevenon A. Visual deficiency and scoliosis. Eur Spine J 2001; 26(1):48-52.

11. Marques MM, Neto JC, Oliveira NML, Oliveira CCE, Ruas G, Shimano SGN. Efeitos da fisioterapia aquática na deficiência visual: relato de caso. RevNeurocienc 2015; 23(1):136-142.

12. Monteiro-Fernández N, Serra-Rexach JA. Role of exercise on sarcopenia in the elderly. Eur J Phys Rehabil Med 2013; 49(1):131-143.

13. Martins NA, Grasiely FB. La discapacidad visual y lapráctica de lasactividades físicas. EFDeportes 2018; 23:247.

14. Cintra VM. Efeitos de um protocolo adaptado de pilates sobre o equilíbrio em deficientes visuais. [Monografia]. Uberaba (MG): Universidade Federal do Triângulo Mineiro; 2018.

15. Cárdenas-Urquizo W. Efeitos de um protocolo adaptado de pilates em deficientes visuais. [Dissertação de Mestrado - Programa de PósGraduação em Fisioterapia]. Uberaba (MG): Universidade Federal do Triângulo Mineiro; 2018.

16. Vilela IC. Efeitos de um protocolo adaptado de pilates na postura e na qualidade de vida de deficientes visuais do instituto dos cegos do Brasil central. [Monografia]. Uberaba (MG): Universidade Federal do Triângulo Mineiro; 2018.

17. Pereira BC, Medalha CC. Avaliação postural por fotometria em pacientes hemiplégicos. ConScientiae Saúde2008; 7(1):35-42.

18. lunes $\mathrm{DH}$. Análise quantitativa do tratamento da escoliose idiopática com - método Klapp por meio da biofotogrametria computadorizada. RevBrasFisioter 2010; 14(2):133-40.

19. Fleck MPA. O instrumento de avaliação de qualidade de vida da Organização Mundial da Saúde (WHOQOL-100): características e perspectivas. CienSaude Colet;2000; 5(1):33-38.

20. Duarte M, Ferreira EA, Maldonado EP Freitas AZ. (2005) Documentação sobre o SAPO - Software para avaliação postural.

21. Greve J, Alonso A, Bordini Ana C, Camanho GL. Correlation between body mass index and postural balance. Clinics 2007; 62(6):717-720.

22. Barela JA. Estratégias de controle em movimentos complexos: ciclo percepção-ação no controle postural. Rev Paul EducFís [Internet] 2016; 3:79-8.

23. Sanchez HM, Barreto RR, Baraúna MA, Canto RST, Morais EG Avaliação postural de indivíduos portadores de deficiência visual através da biofotogrametria computadorizada. FisioterMov 2008; 21(2):11-20.

24. Ferreira EAG. Postura e controle postural: desenvolvimento e aplicação de método quantitativo de avaliação postural. [Tese de Doutorado]. São Paulo (SP): Faculdade de Medicina da Universidade de São Paulo; 2005

25. Reboucas CBA Araújo MM, Braga FC, Fernandes GT Costa SC. Evaluation of quality of life of visually impaired. Rev Bras Enferm [Internet] 2016; 69(1):64-70.

26. Awasthi S, Agnihotri K, Singh U, Thakur S, Chandra H. Determinants of health related quality of life in school-going adolescents in Northern India. Indian J Pediatr 2011; 78(5):555-61.

27. Awasthi S, Agnihotri K, Chandra H, Singh U, Thakur S. Assessment of Health-Related Quality of Life in school-going adolescents: validation of PedsQL instrument and comparison with WHOQOL-BREF. NatlMed $J$ India 2012; 25(2):74-9.

28. Andrade IF, Ely V. Assessment method of accessibility conditions: how to make public buildings accessible? Work 2012; 41(1):3774-3780.

\section{Conclusão}


29. Othero MB, Ayres JRCM. Necessidades de saúde da pessoa com deficiência: a perspectiva dos sujeitos por meio de histórias de vida. Interface 2012; 16(40):219-33.
30. Haegele J, Famelia R, Lee J. Health-related quality of life, physical activity, and sedentary behavior of adults with visual impairments. Disabil Rehabil 2017; 39(22):2269-2276. 